\title{
Emergence of reliable spike patterns in models of CAI cells contacted by unreliable synapses Adam Buntaine $^{1}$, Nadia Corral-Frias ${ }^{2}$ and Jean-Marc Fellous*1,2,3
}

\author{
Address: ${ }^{1}$ Department of Biomedical Engineering, Duke University, Durham, NC 27708, USA, 2Program in Neuroscience, University of Arizona, \\ Tucson, AZ 85024, USA and ${ }^{3}$ Department of Psychology, University of Arizona, Tucson AZ 85024, USA \\ Email: Jean-Marc Fellous* - Fellous@email.arizona.edu \\ * Corresponding author
}

from Sixteenth Annual Computational Neuroscience Meeting: CNS*2007

Toronto, Canada. 7-12 July 2007

Published: 6 July 2007

BMC Neuroscience 2007, 8(Suppl 2):P7| doi:|0.I |86/|47|-2202-8-S2-P7|

(C) 2007 Buntaine et al; licensee BioMed Central Ltd.

Excitatory synapses onto CA1 pyramidal cells fail four times out of five on average, yet the firing of CA1 neuron is elicited at specific phases of the EEG theta cycle with a high degree of precision when a rat is traversing a place field.

We use a multicompartmental biophysical model of several reconstructed CA1 cells, and a model of a stochastic glutamatergic synapse that includes facilitation and depression to study the conditions and properties for reliable and precise CA1 firing. The model of the synapse is tightly constrained by experimental data obtained with minimal stimulations in vitro. Synapses are presynaptically stimulated with CA3/entorhinal spike trains that have been recorded in vivo in the behaving rat.

We report that under those conditions, CA1 pyramidal cells are capable of generating precise spike patterns that are theta-modulated. The precise timing of the pattern depends mainly on either the recruitment of high initial probability synapses, or on the recruitment of weaker perisomatic synapses receiving fast bursts of 1-3 presynaptic action potentials. The patterns generated are robust to noise, and contain a marked theta-frequency component, even though the input spikes are not coherent at any particular frequency. We also report that spike patterns may include gamma-like frequency components in part due to the synaptic dynamics, and to the presence of fast bursts in the presynaptic inputs.
We conclude that even though afferent synapses are unreliable, CA1 pyramidal cells are able to generate precisely timed patterns of spiking that mimic those that are reported in vivo. Our model further predicts that about 400 presynaptic cells are involved in the firing of a single CA1 pyramidal cell when the rat traverses a place field. 